\title{
FUNDAMENTAL SOLUTIONS AND SURFACE DISTRIBUTIONS
}

\author{
by R. A. ADAMS and G. F. ROACH ${ }^{1}$
}

(Received 22nd January 1968)

\section{Introduction}

When studying the solutions of elliptic boundary value problems in a bounded, smoothly bounded domain $D \subset R_{n}$ we often encounter the formula

$$
\int_{\partial D}\left\{u(x) \frac{\partial}{\partial n_{x}} \gamma(x, y)-\gamma(x, y) \frac{\partial}{\partial n_{x}} u(x)\right\} d S_{x}= \begin{cases}u(y) & \text { if } y \in D \\ \frac{1}{2} u(y) & \text { if } y \in \partial D \\ 0 & \text { if } y \notin D\end{cases}
$$

where $u(x) \in C^{2}(D) \cap C^{\prime}(\bar{D})$ is a solution of the second order self-adjoint elliptic equation

$$
L u(x) \equiv\left(\Delta \pm k^{2}\right) u(x)=0, \quad x \in D
$$

and $\frac{\partial}{\partial n_{x}}$ denotes differentiation along the inward normal to $\partial D$ at $x \in \partial D$. $\gamma(x, y)$ is a fundamental solution of (2), and as such has at $x=y$ a singularity described by

$$
\gamma(x, y) \sim \begin{cases}-\frac{1}{2 \pi} \log |x-y| & \text { if } n=2, \\ \frac{1}{2 n-4} \Gamma\left(\frac{n}{2}\right) \pi^{-n / 2}|x-y|^{2-n} & \text { if } n \geqq 3 .\end{cases}
$$

The results (1a) and (1c) can be obtained in a straightforward way by applying Green's Theorem to $u(x)$ and any fundamental solution which is defined in a sufficiently large domain. However the result $(1 b)$ is neither as obvious nor as easily obtained as is generally claimed in textbooks, though, as we shall see, it is true in the sense of the theory of distributions for fundamental solutions which, apart from a singularity of type (3) at $x=y$, are regular in $\bar{D} \times \bar{D}$. For other choices of the fundamental solution (e.g. the Dirichlet Green's function) not satisfying this restriction, $(1 b)$ is meaningless unless a suitable definition can be given for the left hand side. In this paper we shall establish $(1 b)$ for fundamental solutions having the required behaviour in $\bar{D} \times \bar{D}$ and shall show that when a maximum principle is available $\left(L=\Delta-k^{2}\right.$, $k^{2} \geqq 0$ ) $(1 b)$ can be made meaningful in the distributional sense for the Dirichlet Green's function of $L$ and $D$. It is sufficient to demonstrate this for $L=\Delta$.

1 Now at the Department of Mathematics, University of Strathclyde. 
1. For convenience we restrict ourselves to the case $n=2$. Results for $n \geqq 3$ follow in a similar manner. Fix $y \in \partial D$ and let $K_{\varepsilon}$ be the disc of radius $\varepsilon$ centred at $y$. Let $S_{\varepsilon}=\partial K_{\varepsilon} \cap D$. Applying Green's Theorem to $u(x)$ and $\gamma(x, y)$ in $D-K_{\varepsilon}$ and performing a simple residue calculation, noting that on $S_{\varepsilon}$ we have

$$
\gamma(x, y) \sim-\frac{1}{2 \pi} \log \varepsilon, \frac{\partial}{\partial n_{x}}=\frac{\partial}{\partial \varepsilon}
$$

and $d S_{x}=\varepsilon d \theta(0 \leqq \theta \leqq \pi)$, we obtain the result

$$
\lim _{\varepsilon \rightarrow 0} \int_{\partial D-K_{\varepsilon}}\left\{u(x) \frac{\partial}{\partial n_{x}} \gamma(x, y)-\gamma(x, y) \frac{\partial}{\partial n_{x}} u(x)\right\} d S_{x}=\frac{1}{2} u(y) .
$$

Therefore to establish $(1 b)$ it suffices to show that

$$
\lim _{\varepsilon \rightarrow 0} \int_{\partial D \cap K_{\varepsilon}}\left\{\frac{\partial}{\partial n_{x}} \gamma(x, y)-\gamma(x, y) \frac{\partial}{\partial n_{x}} u(x)\right\} d S_{x}=0 .
$$

Since $\partial D$ is smooth, we have in a neighbourhood of $y, d S_{x} \cong d r$ where $r=|x-y|$. Since $\frac{\partial}{\partial n_{x}} u(x)$ is continuous in $\bar{D}$ it follows that

$$
\lim _{\varepsilon \rightarrow 0} \int_{\partial D \cap K_{\varepsilon}} \gamma(x, y) \frac{\partial}{\partial n_{x}} u(x) d S_{x}=\lim _{\varepsilon \rightarrow 0} \frac{-1}{\pi} \frac{\partial}{\partial n_{y}} u(y) \int_{0}^{\varepsilon} \log r d r=0 .
$$

Finally, let $r(x)$ denote the radius of the circle $C_{x}$ through $x$ and $y$ which is tangent to $\partial D$ at $x$. Since $\partial D$ is smooth, $r(x) \rightarrow R$, the radius of the osculating circle for $\partial D$ at $y$, as $x \rightarrow y$. Thus for $\varepsilon$ small enough and $x \in \partial D \cap K_{\varepsilon}$ it follows that $r(x) \geqq \frac{1}{2} R$. If $\alpha$ is the angle between the vector from $y$ to $x$ and the inward normal $n_{x}$ to $\partial D$ at $x$ we have, since $\left|n_{x}\right|=1$ and $\left|\nabla_{x}\right| x-y||=1$, that

$$
\left|\frac{\partial}{\partial n_{x}} \gamma(x, y)\right| \sim \frac{1}{2 \pi}\left|n_{x} \cdot \nabla_{x} \log \right| x-y||=\frac{1}{2 \pi|x-y|}|\cos \alpha| .
$$

By the geometry of the circle $C_{x}$ it follows that

$$
\frac{|\cos \alpha|}{|x-y|}=\frac{1}{2 r(x)} \leqq \frac{1}{R}
$$

and hence we have at once that

$$
\lim _{\varepsilon \rightarrow 0} \int_{\partial D \cap K_{\varepsilon}} u(x) \frac{\partial}{\partial n_{x}} \gamma(x, y) d S_{x}=0
$$

This completes the proof of (4) and so of (1b).

2. The harmonic Green's function, $G(x, y)$, of $D$ is defined for $x \in \bar{D}$, $y \notin \partial D$ by $G(x, y)=\gamma(x, y)+w(x, y)$, where $\gamma(x, y)$ is the function on the right hand side of (3) and

$$
\begin{aligned}
\Delta_{x} w(x, y) & =0, & & x \in D, \\
w(x, y) & =-\gamma(x, y), & & x \in \partial D
\end{aligned}
$$


$G(x, y)$ is positive for $x, y \in D(1, \mathrm{p} .262)$. Since $G(x, y)$ is not properly defined for $y \in \partial D$, the appropriate form of $(1 b)$, namely

$$
\int_{\partial D} u(x) \frac{\partial}{\partial n_{x}} G(x, y) d S_{x}=\frac{1}{2} u(y), \quad y \in \partial D
$$

requires interpretation.

To this end let $G_{\varepsilon}(x, y)$ be the harmonic Green's function for the region $D_{\varepsilon}=D \cap K_{\varepsilon}$. Applying $(1 b)$ over $D$ with $\gamma(x, y)=G_{\varepsilon}(x, y)$, we obtain

$$
\int_{\partial D} u(x) \frac{\partial}{\partial n_{x}} G_{\varepsilon}(x, y) d S_{x}-\int_{\partial D \cap K_{\varepsilon}} G_{z}(x, y) \frac{\partial}{\partial n_{x}} u(x) d S_{x}=\frac{1}{2} u(y) .
$$

Fix $\varepsilon_{0}$. For $\varepsilon<\varepsilon_{0}, G_{\varepsilon_{0}}(x, y)-G_{\varepsilon}(x, y)$ is harmonic in $D_{\varepsilon}$ and non-negative on $\partial D_{\varepsilon}$. By the maximum principle it is non-negative in $D_{\varepsilon}$.

Thus

$$
\left|\int_{\partial D \cap K_{\varepsilon}} G_{\varepsilon}(x, y) \frac{\partial}{\partial n_{x}} u(x) d S_{x}\right| \leqq \text { const. } \int_{\partial D \cap K_{\varepsilon}} G_{e_{0}}(x, y) d S_{x} .
$$

As in (5) above the right hand side tends to zero with $\varepsilon$. Hence

$$
\lim _{\varepsilon \rightarrow 0} \int_{\partial D} u(x) \frac{\partial}{\partial n_{x}} G_{\varepsilon}(x, y) d S_{x}=\frac{1}{2} u(y), \quad y \in \partial D .
$$

This shows that in (6) we may interpret $\frac{\partial}{\partial n_{x}} G(x, y)$ as the limit in the distribution sense as $\varepsilon \rightarrow 0$ of the well defined functions $\frac{\partial}{\partial n_{x}} G_{\varepsilon}(x, y)(2$, Chapter $2 ; 3)$.

\section{REFERENCES}

(1) R. Courant and D. Hilbert, Methods of Mathematical Physics, Vol. II (Interscience, New York, 1962).

(2) I. M. Gel'fand and G. E. ShILov, Generalised Functions, Vol. I (Academic Press, 1964).

(3) L. Schwartz, Théorie des distributions (Hermann et Cié, Paris, 1950).

Department of Mathematics

The UNiversity of British Columbia 